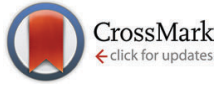

Cite this: Phys. Chem. Chem. Phys., 2014, 16, 25221

Received 12th September 2014 Accepted 15th October 2014

DOI: $10.1039 / c 4 c p 04108 a$

www.rsc.org/pccp

\title{
A perspective on chemistry in transient plasma from broadband rotational spectroscopy
}

\author{
Daniel P. Zaleski, Susanna L. Stephens and Nicholas R. Walker* \\ Broadband rotational spectroscopy provides a new method by which plasma chemistry can be explored. \\ Molecules and complexes form when precursors within an expanding gas sample are allowed to interact \\ with plasma generated by an electrical discharge or laser vaporisation of a solid. It is thus possible to \\ selectively generate specific molecules or complexes for study through a careful choice of appropriate \\ precursors. It is also possible to survey an extensive range of the products formed under a given set of \\ initial conditions in an approach termed "broadband reaction screening". Broadband rotational \\ spectroscopy provides an opportunity to simultaneously monitor the transitions of many different \\ chemical products and this allows broader details of reaction pathways to be inferred. This Perspective \\ will describe various experimental approaches and review recent works that have applied broadband \\ rotational spectroscopy to study molecules and complexes generated (in whole or in part) through \\ chemistry occurring within transient plasma.
}

\section{Introduction}

The earliest experiments in microwave spectroscopy probed chemical samples under thermal equilibrium and demonstrated the value of pure rotational spectroscopy for the characterisation of molecular structure. ${ }^{1-5}$ The subsequent development of the Balle-Flygare Fourier transform microwave (BF-FTMW) spectrometer $^{6}$ introduced a step-change in the capabilities of spectrometers operating at microwave frequencies. It became possible to perform precise and selective measurements on weakly-bound and pre-reactive complexes. $^{7-9}$ The chirped-pulse FTMW (CP-FTMW) spectrometer constructed by Pate and co-workers ${ }^{10}$ represents another major advance in the methods and instrumentation used for microwave spectroscopy as described in a recent review article. ${ }^{11}$

Researchers are now exploring whether broadband rotational spectroscopy might find wider applications in chemical analysis. For various reasons explained recently, it is likely that general applications of broadband rotational spectroscopy for chemical analysis will be most viable at mm-wave frequencies near $200 \mathrm{GHz} .{ }^{12-14}$ However, it is also possible to provide insight into the nature of selected chemical environments through experiments performed at centimetre wavelengths. The low temperatures present in a gas sample undergoing supersonic expansion $(\sim 2 \mathrm{~K})$ allow the sensitive detection of many chemical products between 2 and 40 gigahertz in frequency. Many researchers already have access to broadband spectrometers operating within this range. ${ }^{15-19}$ Where these are configured

School of Chemistry, Bedson Building, Newcastle University, Newcastle upon Tyne, Tyne and Wear, NE1 7RU, UK.E-mail: nick.walker@newcastle.ac.uk to allow an electrical discharge ${ }^{20}$ or focussed laser pulse ${ }^{21}$ to interact with the expanding sample, it becomes possible to isolate and observe chemical products generated within the environment of a transient plasma. Aside from the opportunity to study the molecular physics of products generated in this way, experiments expose the environment in plasma to broader characterisation. Details of reaction pathways within plasma can be inferred from observations of the range and characteristics of chemicals that are present. This technique is known as broadband reaction screening, and in many ways is similar in concept to the well-known Urey-Miller "spark" experiments in prebiotic chemistry. $^{22,23}$

This Perspective will review how broadband rotational spectroscopy has recently been employed to characterise chemical products generated entirely or partially within transient plasma accompanying laser vaporisation of a solid material or electrical discharge through a gas sample. In some cases, experiments were initially motivated by questions in fundamental molecular physics but observations also provided broader insight into chemical reactions within plasma. In other studies, the primary motivation was the characterisation of a wide range of synthetic pathways under specified initial conditions of chemical composition and plasma temperature. Because the samples being studied often contain multiple distinct molecular species (e.g. several rotational conformers and structural isomers), the high spectral resolution of these experiments demonstrate that broadband rotational spectroscopy can provide insight into the nature of extreme chemical environments that is not typically available through other techniques due to spectral overlap. They also provide the opportunity to develop and test computational and other methods for interpreting spectra that may ultimately find 
broader applications in chemical analysis. Finally, experiments have revealed pathways allowing the generation of molecules that cannot readily be synthesised for spectroscopic study in the laboratory by other methods.

\section{Experimental}

Fourier transform microwave (FTMW) spectroscopy employs a sequence of events that comprises a gas pulse, microwave pulse, detection of the spontaneous coherent emission (the free induction decay, or FID) and Fourier transform of the detected radiation. FTMW spectroscopy is physically analogous to FT-NMR spectroscopy but distinct in the sense that it requires interaction of the radiation field with an electric rather than magnetic dipole. The behaviour of the molecular ensemble over time is fundamentally described by the Bloch equations ${ }^{24}$ that are also used to underpin the physical description of FT-NMR experiments.

Fourier transform microwave spectroscopy became practicable for research in chemistry during the late 1970's and was pioneered by Balle and Flygare. ${ }^{6}$ Electronics technology provided digitisers of kilohertz sampling rates which were insufficiently fast to permit FIDs of many gigahertz (i.e. microwave frequencies) to be analysed. Fortunately, heterodyning methods (where the FID is mixed down against a carrier wave) made it possible to view any short section of spectrum (typically less than $1 \mathrm{MHz}$ ) within a tuneable range of the spectrometer of $12 \mathrm{GHz}$. As a consequence of the narrow bandwidth of individual measurements, it was a logical step to enhance the sensitivity of this technique through the inclusion of a tuneable microwave cavity. The Balle-Flygare FTMW spectrometer has since been used widely to characterise molecules and complexes that are isolated at the cold temperatures achieved in supersonically-expanding gas samples. One feature of this spectrometer is the use of a short duration (typically $\sim 1 \mu \mathrm{s})$ microwave pulse from a fixed-frequency source to achieve initial polarisation of the molecular sample. The finite frequency bandwidth of this pulse is determined by the pulse duration as consistent with the Heisenberg uncertainty principle. The bandwidth of an individual measurement thus cannot be changed independent of the duration of the excitation pulse. It is an associated disadvantage that the power of the excitation pulse cannot be optimised for a specific molecule across the full bandwidth of a single measurement.

The general principles of CP-FTMW spectroscopy have been described by Pate and co-workers. ${ }^{10}$ This method exploits recent advances in technology made by the telecommunications industry. It is now possible to directly digitise FIDs exceeding $1 \mathrm{GHz}$ in frequency. In addition, the transform-limited pulse used to polarise the molecular ensemble in the Balle-Flygare FTMW spectrometer can be replaced by a "chirped" pulse that is rapidly (within $\sim 1 \mu \mathrm{s}$ ) swept across the entire frequency bandwidth of a measurement. Thus, broadband spectra spanning many gigahertz in frequency can be recorded efficiently in a single measurement. Excitation pulse duration, bandwidth and power are independent variables in CP-FTMW spectroscopy. In general, CP-FTMW spectrometers are constructed with the specific aim of performing broadband measurements and thus do not contain any microwave cavity. The technique requires that the duration of the chirped pulse is significantly shorter than the timescale of the subsequent molecular emission, detected as the free induction decay (FID) of the polarisation, and is typically selected to be $\sim 1 \mu \mathrm{s}$. The linewidth of a single transition is determined by the timescale of the FID which is typically of the order of $20 \mu \mathrm{s}$. High-power travelling wave tube amplifiers (TWTA) are available and allow molecules to be efficiently polarised and probed across a high bandwidth $(\sim 10 \mathrm{GHz})$ in a single measurement. Additional to high bandwidth, a further advantage of CP-FTMW spectroscopy is that the intensities of transitions recorded and displayed by the spectrometer authentically reflect the intrinsic properties of the molecules and sample across the probed bandwidth. The relative intensities of transitions are directly related to relative dipole moment components and transition dipole matrix elements. The value of intensity measurements that correctly reflect the intrinsic properties of the molecules and sample probed is most quickly realised when searching for isotopic species in dense broadband datasets. For example, the spectrum of a species containing one ${ }^{13} \mathrm{C}$ in natural abundance would appear with $1 \%$ of the intensity of the normal species containing ${ }^{12} \mathrm{C}$. Furthermore, having reliable relative intensities allows for the quantitative analysis of samples where multiple species are present.

The procedure used to generate metal-containing molecules for study by CP-FTMW spectroscopy is essentially the same as that previously employed in some Balle-Flygare FTMW spectrometers. A gaseous sample containing a mixture of precursors is pulsed into an evacuated chamber through a nozzle with an orifice diameter of less than $1 \mathrm{~mm}$. The sample undergoes supersonic expansion shortly after passing through the plasma initiated by an electrical discharge or focussed laser pulse. For the purposes of this Perspective, it will be assumed that the latter is focussed onto a solid target from which material is vaporised by the laser pulse. When configured for CP-FTMW spectrometers, electrical discharge and laser vaporisation sources are usually bespoke to meet local requirements and constraints. The former typically presents the advantage of being somewhat easier to design and configure within constraints imposed by the wider operation of the instrument. The latter allows for more consistent reproduction of experimental conditions given that an experiment involves repeating measurements over many averaging cycles. Experiments have shown that vaporisation, heating and ionisation of atoms ejected from a solid by a high energy, focussed laser pulse can occur on a timescale of picoseconds. ${ }^{25}$ Nd:YAG lasers operating on the second or third harmonics (532 or $355 \mathrm{~nm}$ respectively) are often used for such experiments and the pulse from such a laser is typically between 5 and 15 nanoseconds in duration. Such a laser pulse thus continues to interact with species within the nascent plasma subsequent to the initial vaporisation of material from the solid. By definition, plasma contains a highly-excited mixture of atoms, molecules, electrons and photons. The atoms and molecules may be neutral, singly- or multiply-charged. Components of plasma interact to yield chemical products that depend on the reactants and plasma conditions. 
Many alternative microwave circuits could be used in CP-FTMW spectroscopy and schemes employed vary between laboratories according to individual needs. During experiments conducted in our group, and following supersonic expansion of the sample prepared as described above, a chirped pulse of microwave radiation spanning $\mathbf{7 . 0}-\mathbf{1 8 . 5} \mathrm{GHz}$ induces macroscopic rotational polarisation of molecules within the sample on resonance with molecular rotational transitions. The polarising pulse is prepared by mixing a $12-0.5 \mathrm{GHz}$ chirped pulse from an arbitrary waveform generator (AWG) against the filtered output of a $19 \mathrm{GHz}$ reference supplied by a phase-locked dielectric resonant oscillator (PDRO). The additional unwanted sideband is removed when the excitation pulse passes through a 6.5-18.5 GHz bandpass filter. The microwave pulse is input to a pulsed $300 \mathrm{~W}$ travelling wave tube amplifier (TWTA) and then transmitted toward the expanding gas jet via a broadband horn antenna whereas the molecular FID is received at a second broadband horn antenna (Fig. 1). The resulting FID from 7.0-18.5 GHz is amplified by a low noise amplifier (LNA, $55 \mathrm{~dB}$ gain, $2 \mathrm{~dB}$ noise figure) and mixed down against the $19 \mathrm{GHz}$ reference frequency before passing through a $12.5 \mathrm{GHz}$ lowpass filter to allow digitisation and processing to be performed between 0.5 and $12.0 \mathrm{GHz}$ on a $25 \mathrm{Gs} \mathrm{s}^{-1}$ digitiser. The back-end is protected from the high power TWTA by a switch. Measurements are repeated and averaged to improve the signal/noise ratio of observed transitions. Multiple FIDs are recorded following each sample injection cycle to achieve a reduction in the time necessary to accumulate measurements and hence achieve a reduction in total sample consumption. The accumulated FID's are first co-added in the time domain and then the average is calculated. Finally, the FID is Fourier transformed to obtain the frequency domain spectrum. The digital design has excellent phase stability (necessary for

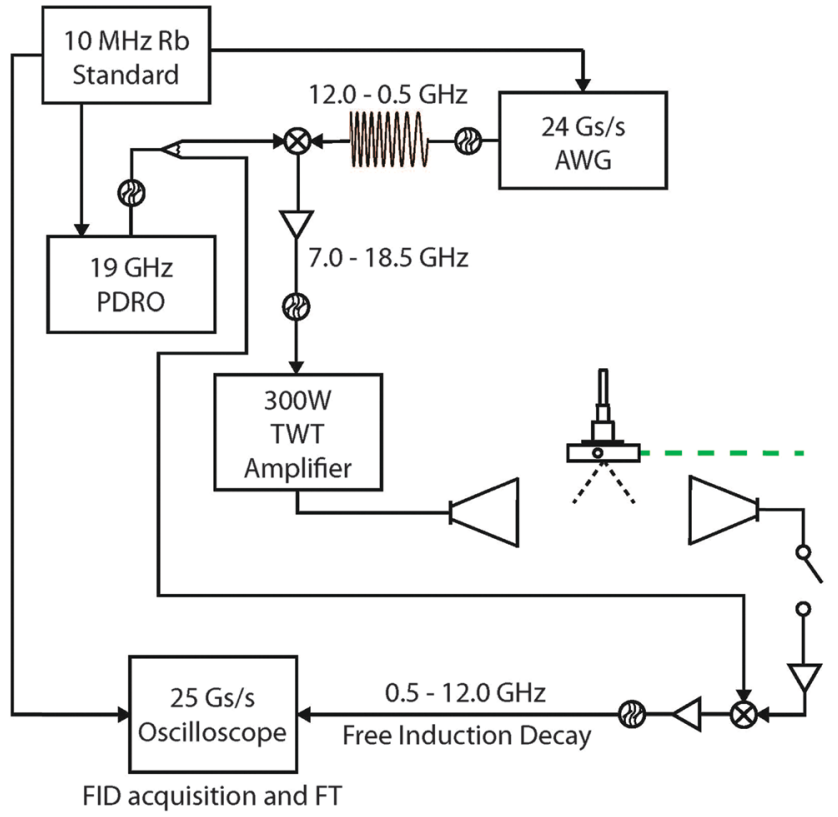

Fig. 1 Schematic of the Newcastle CP-FTMW spectrometer. The green dashed line represents the laser beam path. averaging over an extended period) and it is now routine to collect $>1 \mathrm{M}$ signal acquisitions. ${ }^{26-29}$ Accurate frequency measurements are obtained by locking to an external reference source accurate to 1 part in $10^{11}$.

Propagation of the molecular beam is perpendicular to the orientation of the detecting horn such that each transition is observed as a single peak with full-width at half maximum of FWHM $\cong 150 \mathrm{kHz}$ after application of a Kaiser-Bessel digital filter to improve baseline resolution. Detailed specifications of a CP-FTMW spectrometer used for the experiments employing laser vaporisation are provided in ref. 30. A description of a CP-FTMW spectrometer configured with an electrical discharge source is available in ref. 15 and 20.

\section{Results}

\section{Exploiting plasma chemistry to generate novel molecules and complexes for study by broadband rotational spectroscopy}

Molecules introduced into a plasma environment may undergo extensive fragmentation and/or chemical reaction. It is well known that such processes can provide a convenient method for generating molecules and clusters that are of considerable fundamental interest. Moreover, chemical products formed within plasma can be entrained within a buffer gas, stabilised within the cold environment of a supersonic expansion and thus prepared for interrogation by any of a wide range of spectroscopic techniques. CP-FTMW spectroscopy is a comparatively recent addition to a long list of spectroscopic techniques that have been applied to study molecules and complexes generated in this way. ${ }^{31-34}$

Fourier transform microwave spectroscopy (using either BF- or CP-FTMW spectrometers) can generally be performed at high resolution such that transitions are recorded with typical FWHM (full width at half maximum) of less than $150 \mathrm{kHz}$. This high resolution greatly assists the unambiguous assignment of spectra to carrier molecules and facilitates highly accurate determination of molecular geometries. Many complexes were generated and studied using Balle-Flygare FTMW spectrometers equipped with laser vaporisation or electrical discharge sources before the introduction of CP-FTMW spectroscopy. These experiments permitted new insight into the molecular geometry of neutral, metal-containing molecules. ${ }^{35-39}$ An implication of the narrow ( $\sim 1 \mathrm{MHz})$ measurement bandwidth of a Balle-Flygare FTMW spectrometer is that a researcher must usually select a specific molecule to serve as the target of a study, then aim to predict the transition frequencies of that molecule with reasonable accuracy before starting an experiment. CP-FTMW spectroscopy allows transitions to be simultaneously measured across a greater, typically $12 \mathrm{GHz}$, bandwidth.

An example of results ${ }^{40}$ that have recently been acquired using a CP-FTMW spectrometer is shown in Fig. 2. The gaseous sample prepared in advance of this experiment contains very small amounts of $\mathrm{CF}_{3} \mathrm{I}$, $\mathrm{CO}$ as well as argon in high concentration. The mixture is passed over the surface of a silver rod from which metal is vaporised by the focussed pulse from a 


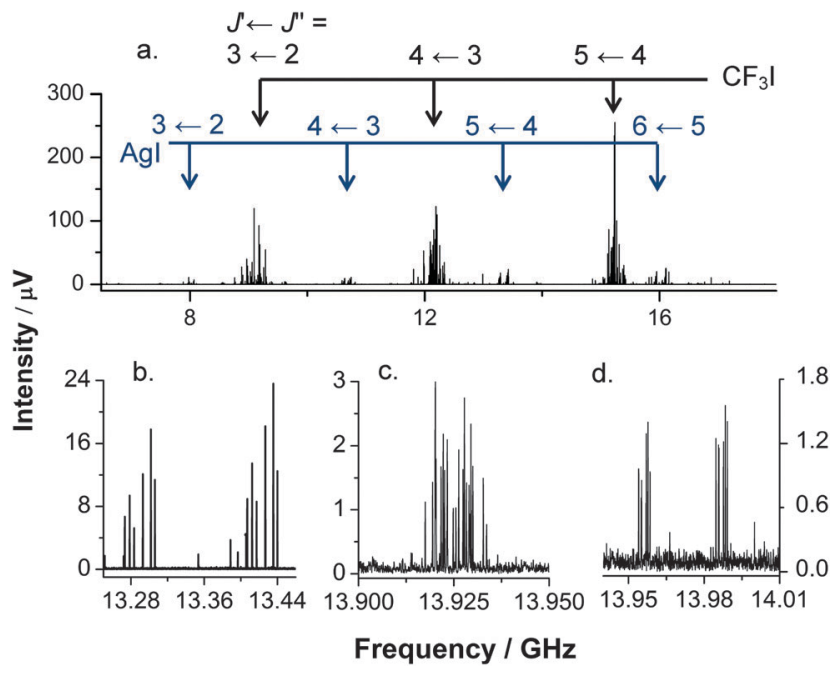

Fig. 2 Data originally reported in ref. 40 and resulting from averaging 146k FIDs (5 hours of real time). (a) Broadband spectrum obtained when probing sample containing small percentages of $\mathrm{CF}_{3} \mathrm{l}, \mathrm{CO}$ and $\mathrm{Ag}$ in $98 \%$ argon. (b) ( $\times 12)$ magnification of the intensity axis reveals spectrum of Agl. (c) and (d) $(\times 100)$ magnifications of the intensity axis reveal the spectra of $\mathrm{CF}_{3} \mid \ldots \mathrm{CO}$ and $\mathrm{OC} \ldots \mathrm{Agl}$ respectively.

Nd:YAG laser. Transitions in the resulting spectrum arise from molecules and complexes that may be (i) introduced into the sample prepared initially; (ii) formed within the cold environment of the expanding jet from components of the sample prepared initially or (iii) formed from fragments of precursor molecules that are generated within the plasma that accompanies the laser pulse. Given signals of sufficiently high intensity, performing a measurement with the laser switched off provides an easy check of whether or not a molecule fits into the third of these categories. Where laser- or plasma-induced processes are crucial for the formation of a complex, it will not be observed with the laser switched off. The same on-off test can be performed in experiments that exploit an electrical discharge to learn whether a selected transition is dependent upon chemistry initiated by the discharge.

The most intense transitions in the $12 \mathrm{GHz}$ broadband spectrum of Fig. 2, panel (a) are readily assigned to the $\mathrm{CF}_{3} I$ monomer that is abundant in the initially-prepared gas sample. These remain present when the laser is switched off and are observed to be slightly reduced in intensity when the laser is on. Some observed transitions assign to a complex ${ }^{41}$ of $\mathrm{CF}_{3} \mathrm{I}$ with $\mathrm{CO}$ that forms from the precursors, $\mathrm{CF}_{3} \mathrm{I}$ and $\mathrm{CO}$, within the supersonic expansion. These are also observed with lower intensity when the laser is on. Other observed features confirm that some $\mathrm{CF}_{3} \mathrm{I}$ molecules undergo fragmentation when the laser is on to yield fragments that subsequently recombine to form new chemical species. ${ }^{42}$ Such transitions are not observed unless the laser is on. The most intense transitions in this category assign to the diatomic molecules, AgI, AgF and IF, which can themselves bind to other molecules to form complexes. Transitions of OC..AgI, indicated in Fig. 2(d), recently allowed characterisation of the geometry of this complex for the first time. ${ }^{40}$ Other experiments by our group have focussed on the complexes formed between $\mathrm{H}_{2} \mathrm{~S}, \mathrm{C}_{2} \mathrm{H}_{2}$ or $\mathrm{C}_{2} \mathrm{H}_{4}$ and $\mathrm{MX}$ (where $\mathrm{M}$ is either $\mathrm{Cu}$ or $\mathrm{Ag}$ and $\mathrm{X}$ is $\mathrm{Cl}$ or I) ${ }^{43-45}$ As with previous studies that exploited Balle-Flygare FTMW spectrometers, these experiments involved the selection of precursors appropriate to the generation of a chemical product that was specifically targeted for study in advance.

A number of groups have performed similar experiments using CP-FTMW spectrometers to study products generated through either laser vaporisation or electrical discharge in recent years. The first example of a CP-FTMW spectrometer configured with a laser ablation source for the generation of metal-containing molecules was described by Grubbs et al. in $2007 .{ }^{18}$ It was shown that the opportunity to record transition intensities accurately reflecting the transition dipole matrix elements is of considerable value in challenging tasks of spectral assignment. The pure rotational spectrum of $\mathrm{SnCl}$ was assigned despite the challenge presented by the open-shell electronic structure of this molecule. ${ }^{46}$ A laser vaporisation source has also been used to transfer biological molecules into the gas phase for study by CP-FTMW spectroscopy. ${ }^{30}$ It is usually desirable to suppress biomolecule fragmentation and/or chemical reactions that yield other products so it is not necessarily favourable to generate plasma during such experiments.

A major advantage of CP-FTMW spectroscopy is the greatlyenhanced opportunity to assign the spectra of novel molecules and complexes that are not amongst the molecular targets initially selected for a study. Novel molecular complexes may be readily identified even where their presence is not anticipated and where accurate predictions of transition frequencies are not available beforehand. An example arises from recent experiments that characterised the geometry of the $\mathrm{C}_{2} \mathrm{H}_{2} \cdots \mathrm{AgCCH}$ complex. ${ }^{47}$ Natural samples of many common metals, including silver, are comprised of more than one naturally-abundant isotope leading to distinctive and characteristic patterns of transitions in spectra. Such distinctive patterns are of considerable value in facilitating the assignment of spectra whose molecular carriers are initially unknown and unpredicted. The patterns arising from nuclear quadrupole coupling can serve a similar purpose. Transitions of $\mathrm{C}_{2} \mathrm{H}_{2} \cdots \mathrm{AgCCH}$ were observed in patterns consistent with assignment to an asymmetric top of $C_{2 \mathrm{v}}$ geometry that contains a silver atom (assigned on the basis of the observed spectra of different isotopologues) very close to the centre of mass. These were initially suspected as assigning to the $\mathrm{C}_{2} \mathrm{H}_{2} \cdots$ AgF complex that had initially been selected as the target of the study. However, subsequent examination of the rotational constants confirmed that the spectrum must instead assign to $\mathrm{C}_{2} \mathrm{H}_{2} \cdots$ AgCCH. The results of this experiment illustrate how the opportunity to survey the entire $12 \mathrm{GHz}$ bandwidth of the spectrometer in a single measurement provides greatly enhanced opportunities to detect and analyse unexpected chemical products that lie outside the initial aims of a study.

It is not always easy to predict which chemical products are likely to result when a mixture of precursors passes through plasma generated by a laser vaporisation or electrical discharge. As a consequence, CP-FTMW spectroscopy provides a new opportunity to identify species that have not been observed before. Different strategies can be adopted. It is possible for the researcher to gain greater insight into the broader chemical 
environment within the plasma itself. This possibility is explored further below.

\section{Screening of products of reactions and fragmentation in plasma by broadband rotational spectroscopy}

As previously noted, the BF- and CP-FTMW designs are complimentary, but the CP-FTMW design is particularly well suited for broadband survey spectra that are performed without reference to initial assumptions about the probed species. Its broadband capability allows easier adjustment and optimisation of sample conditions when the molecular carrier(s) of rotational transitions is/are initially unknown. This capability is quite important for astrochemical applications as many radio astronomy survey spectra ${ }^{48}$ overlap with the frequency coverage of many laboratory-based CP-FTMW spectrometers. The coupling of broadband rotational spectroscopy techniques with broadband spectral surveys from radio astronomy has thus aided in analysing the complex mixtures present in both.

A pulsed DC discharge source can be easily fitted to both cavity-based and broadband FTMW spectrometers. Furthermore, discharge sources have shown utility in producing novel species of astrochemical interest, such as long chain unsaturated alkynes. $^{49,50}$ Pulsed discharge nozzles have also been used to study molecular ions and radicals ${ }^{51-54}$ which were subsequently identified in the interstellar medium (ISM). Additionally, pulsed discharge nozzles have successfully created high energy isomers of molecules, whether they be conformational, like the recently detected trans isomer of methyl formate ${ }^{55,56}$ towards Sagittarius $\mathrm{B} 2(\mathrm{~N})$, or structural isomers like HSCN,${ }^{57}$ a higher energy form of HNCS, also towards Sagittarius B2(N).

CP-FTMW spectrometers have been used to characterise the rich chemistry produced by these pulsed discharge nozzles. The measurement concept of the reaction screening experiments is similar to the previously mentioned Urey-Miller experiments. An abundant interstellar molecule, or combination of molecules, is subjected to the reactive conditions of the discharge source. Many of the species present can be immediately identified if they have been previously recorded. Astrochemical databases such as Splatalogue $^{58}$ and $\mathrm{CDMS}^{59}$ are continuously being developed to make this process as efficient as possible. For species not catalogued in the databases, one method for focusing in on high priority unidentified targets is to directly compare broadband laboratory datasets with the publically available GBT PRIMOS radio astronomy survey spectra of Sagittarius B2(N). ${ }^{48}$

Chemistry initiated by a discharge source and studied within the laboratory tends to yield transitions that overlap in frequency with unassigned transitions in the PRIMOS survey. ${ }^{48}$ Two examples of this are the $E$ - and $Z$-isomers of ethanimine $\left(\mathrm{CH}_{3} \mathrm{CHNH}\right),{ }^{60}$ shown in Fig. 3, and $E$-isomer of cyanomethanimine $(\mathrm{HNCHCN})^{61}$ via discharges of $\mathrm{CH}_{3} \mathrm{CN}$ and $\mathrm{CH}_{3} \mathrm{CN}+\mathrm{NH}_{3}$, respectively. The former was previously suggested as a possible interstellar molecule after the detection of methenamine $\left(\mathrm{CH}_{2} \mathrm{NH}\right)^{62}$ but their spectra were not catalogued and thus eluded detection. Frequencycoincident bands in the two spectra shown in Fig. 3 provided confirmation that ethanimine is present in the interstellar medium. The detection of ethanimine has proven quite fortunate, as the

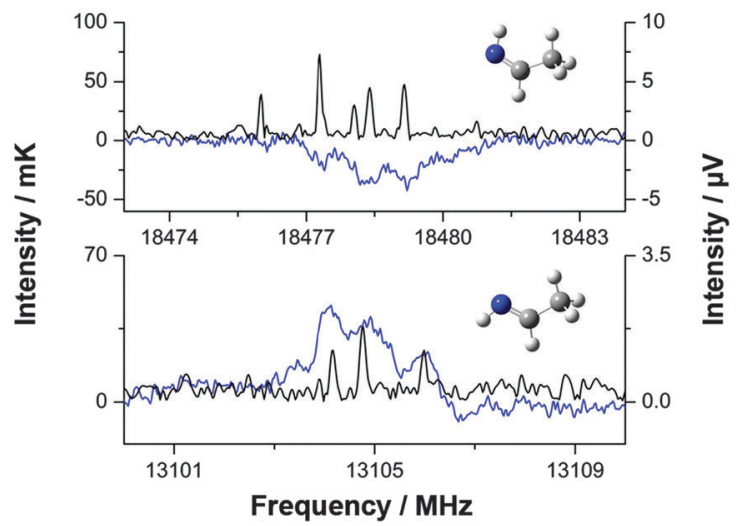

Fig. 3 The black trace is the $6.5-20 \mathrm{GHz}$ chirped-pulse FTMW spectrum of a $2 \mathrm{kV} \mathrm{DC}$ discharge of acetonitrile (laboratory intensity on the right). The blue trace is the GBT PRIMOS survey (intensity on the left). The data for this figure was taken from ref. 60.

related nitriles - $\mathrm{HCN}, \mathrm{CH}_{2} \mathrm{NH}$, and $\mathrm{NH}_{2} \mathrm{CH}_{2} \mathrm{CN}$ - have all been identified in the ISM, ${ }^{63}$ and have been shown experimentally to participate in a modified Strecker route to produce the simplest amino acid, glycine. ${ }^{64}$ The detection of ethanimine suggests a similar route to the production of alanine. Likewise, cyanomethanimine, which is widely considered to be a key intermediate in the possible prebiotic formation of adenine, ${ }^{65-67}$ was also not included in the catalogues. This synthetic route typically requires the condensation, or oligomerisation, of five HCN units; ${ }^{68-71}$ however, the discharge chemistry suggests a possible radical formation route to the first species, cyanomethanimine, also known as the HCN dimer. Adenine is one of the two purine nucleobases, and its prebiotic formation may play a critical role in the RNA-world scenario for the formation of life on Earth. ${ }^{72}$

Due to its utility, the broadband reaction screening technique has also seen success in other areas. Using this technique, two new molecules were recently detected ${ }^{73}$ - HSiNSi and $\mathrm{H}_{3}$ SiNSi after a discharge of mixtures containing silane and either nitrogen or ammonia. Prior to the reaction screening measurements, neither species had been the interest of experimental or theoretical studies. It turns out that these species may be important intermediates, via reactions with the SiN radical, ${ }^{74}$ in chemical vapour deposition (CVD) techniques used to grow films. ${ }^{75-79}$ A similar study investigated the dissociation products of 2,3-dihydrofuran $(2,3-\mathrm{DHF}){ }^{80}$ The unbiased nature of the broadband screening identified 7 distinct chemical products formed as a result of the electrical discharge, as well as conformational isomers of many of the species. One of the most interesting aspects of the study was the observation of formaldehyde. Formaldehyde was proposed to be a side product of 2,3-DHF ring-opening, ${ }^{81,82}$ the other product being propyne which was also observed. The study further speculated that the excess energy from the discharge could cause propyne to dissociate to vinylidenecarbene $\left(\mathrm{C}_{3} \mathrm{H}_{2}\right)$, the rearrangement of which could then explain the detection of cyclopropenylidene $\left(\mathrm{c}-\mathrm{C}_{3} \mathrm{H}_{2}\right)$.

The above results highlight the value of broadband spectral surveys performed without preconceived notions, with regards to experiment or theory, of what to expect. This measurement 


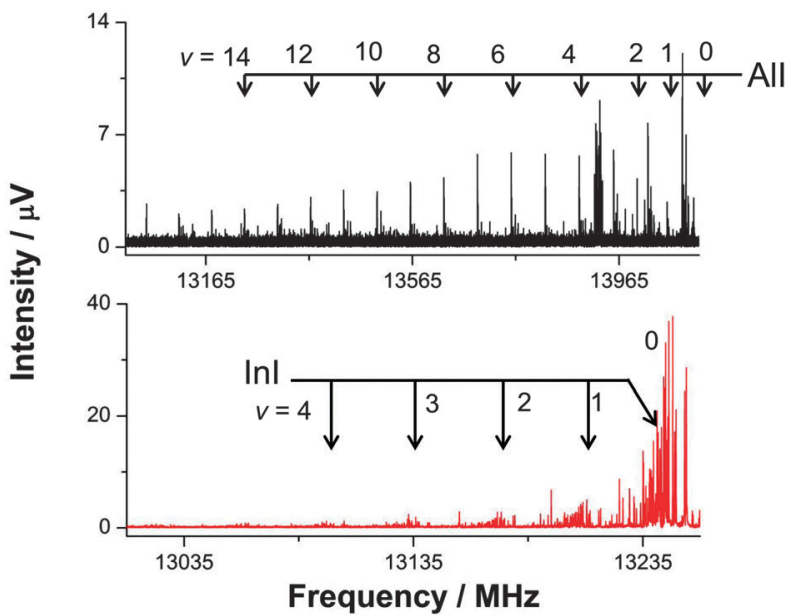

Fig. 4 (top) Broadband spectrum obtained by probing an expanding sample containing small percentages of $\mathrm{CF}_{3} \mathrm{l}, \mathrm{CO}, \mathrm{Al}$ and $98 \%$ argon. Average of $259 \mathrm{k}$ FIDs (9 hours in real time). The $J^{\prime} \leftarrow J^{\prime \prime}=3 \leftarrow 2$ rotational transition is observed for vibrational states between $v=0$ and 14. (bottom) Broadband spectrum obtained by probing an expanding sample containing small percentages of $\mathrm{CH}_{3} \mathrm{l}, \mathrm{CO}, \mathrm{In}$ and $97 \%$ argon. Averaged over $88 \mathrm{k}$ FIDs ( 3 hours in real time). The $J^{\prime} \leftarrow J^{\prime \prime}=6 \leftarrow 5$ rotational transition is displayed for vibrational states between $v=0$ and 4 .

technique is especially powerful when combined with plasma chemistry, where the reaction products may not necessarily be easy to predict.

\section{Rapid detection and measurement of pure rotational spectra of molecules in vibrationally-excited states}

The ability to perform rapid survey spectra allows rotational transitions associated with many different vibrational states to be recorded simultaneously. Vibrational states of SnS between $v=0$ and $v=6$ (inclusive) were observed during a study by Grubbs et al. ${ }^{83}$ Recent experiments by our group allow the assignment of pure rotational spectra of InI in vibrational states between $v=0$ and $v=4$ (Fig. 4). Similar advantages were realised during measurements of pure rotational spectra of AlI between $v=0$ and $v=14$; SnI between $v=0$ and $v=8$; and $\mathrm{PbI}$ between $v=0$ and $v=6 .{ }^{84}$ The patterns observed in broadband rotational spectra are highly distinctive. It is possible to obtain accurate models of potential energy surfaces ${ }^{85}$ from parameters determined for different vibrational states through pure rotational spectroscopy. In respect of such studies, the principal advantage conferred by broadband instruments arises from the speed with which spectroscopic transitions can be identified, assigned and measured. The reduction in the time necessary to perform measurements increases the likelihood that data from pure rotational spectroscopy will be more frequently used for accurate modelling of potential energy surfaces in the future.

\section{Conclusions}

Plasma is the state of matter found abundantly in stars. It is the result of transient, intermittent events such as lightning strikes or meteor impacts in the terrestrial environment and is thus often regarded as an "exotic" state. Commercially, the production of materials by chemical vapour deposition is assisted by plasma chemistry. It is thus important to experimentally-characterise chemistry initiated or mediated by plasma but this is challenging because of the high temperatures involved and the transient nature of intermediates. Spectroscopy has been shown to allow the sensitive detection of a wide range of chemical species by remote methods. ${ }^{86-88}$

The experiments described herein illustrate that broadband rotational spectroscopy presents new opportunities to follow chemical reactions and allows the products of plasma chemistry to be identified. Chemistry initiated by either an electrical discharge or laser vaporisation of a solid material has been the focus of this article. There have been several other, recent applications of broadband rotational spectroscopy to study chemical products generated at high temperatures and these have involved initiation by pyrolysis. Kidwell et al. ${ }^{89}$ recently studied cyclopentadienone, $\mathrm{C}_{5} \mathrm{H}_{4} \mathrm{O}$, generated through flash pyrolysis and probed by broadband rotational spectroscopy. Isotopologues containing ${ }^{13} \mathrm{C}$ were studied in natural abundance allowing a detailed exploration of the molecular structure. A recent Perspective article by Prozument et al. ${ }^{14}$ describes the application of broadband rotational spectroscopy to monitor both the range of chemical products formed following pyrolysis and energy partitioning between different vibrational states. The result is a much more extensive and complete perspective on the chemistry of pyrolysis than was available previously.

New insights into the chemistry occurring in extreme environments will clearly emerge from broadband rotational spectroscopy. The chances that a novel spectrum can be identified following chemical reactions initiated at a high temperature are enhanced by broadband methods. It is not always necessary or useful to select a single "target" molecule for study in advance of experiments. Whilst broadband rotational spectroscopy is restricted to the study of molecules possessing a non-zero electric dipole moment, it can be anticipated that other current constraints will be overcome. The significant challenge associated with the assignment of spectra to their correct molecular carriers will be reduced by greater understanding of the chemistry in plasma environments. The sensitivity of CP-FTMW spectroscopy is directly connected with the speed of digitisers in component instruments and this can be expected to increase over the next 10 years. Finally, computational tools allowing the efficient deconvolution, assignment and analysis of complex spectra are under development. Evidently, broadband rotational spectroscopy will become increasingly useful as a tool for the exploration of plasma chemistry.

\section{Acknowledgements}

The authors thank the European Research Council for postdoctoral fellowships awarded to D.P.Z., S.L.S. and for project funding (CPFTMW-307000). D.P.Z. also thanks Newcastle University for the award of a Faculty of SAgE Research Fellowship. The authors also thank the EPSRC for the award of Research Grant No. EP/G026424/1 in support of various experiments reviewed here. 


\section{References}

1 D. G. Scroggin, J. M. Riveros and E. B. Wilson Jr., J. Chem. Phys., 1974, 60, 1376.

2 E. B. Wilson Jr., Chem. Soc. Rev., 1972, 1, 293.

3 R. G. Ford and R. A. Beaudet, J. Chem. Phys., 1971, 55, 3110.

4 R. W. Kilb, C. C. Lin and E. B. Wilson Jr., J. Chem. Phys., 1957, 26, 1695.

5 E. B. Wilson Jr., Proc. Natl. Acad. Sci. U. S. A., 1957, 43, 816. 6 T. J. Balle and W. H. Flygare, Rev. Sci. Instrum., 1981, 52, 33. 7 G. Columberg and A. Bauder, J. Chem. Phys., 1997, 106, 504. 8 L. W. Buxton, E. J. Campbell and W. H. Flygare, Chem. Phys., 1981, 56, 399.

9 G. T. Fraser, K. R. Leopold, D. D. Nelson Jr., A. Tung and W. Klemperer, J. Chem. Phys., 1984, 80, 3073.

10 G. G. Brown, B. C. Dian, K. O. Douglass, S. M. Geyer, S. T. Shipman and B. H. Pate, Rev. Sci. Instrum., 2008, 79, 053103.

11 M. Schnell, Z. Phys. Chem., 2013, 227, 1.

12 A. L. Steber, B. J. Harris, J. L. Neill and B. H. Pate, J. Mol. Spectrosc., 2012, 280, 3.

13 J. L. Neill, B. J. Harris, A. L. Steber, K. O. Douglass, D. F. Plusquellic and B. H. Pate, Opt. Express, 2013, 21, 19743.

14 K. Prozument, G. B. Park, R. G. Shaver, A. K. Vasiliou, J. M. Oldham, D. E. David, J. S. Muenter, J. F. Stanton, A. G. Suits, G. B. Ellison and R. W. Field, Phys. Chem. Chem. Phys., 2014, 16, 15739.

15 D. P. Zaleski, J. L. Neill, M. T. Muckle, N. A. Seifert, P. B. Carroll, S. L. Widicus Weaver and B. H. Pate, J. Mol. Spectrosc., 2012, 280, 68.

16 S. L. Stephens and N. R. Walker, J. Mol. Spectrosc., 2010, 263, 27.

17 C. Pérez, S. Lobsiger, N. A. Seifert, D. P. Zaleski, B. H. Pate, Z. Kisiel, B. Temelso and G. C. Shields, Chem. Phys. Lett., 2013, 571, 1.

18 G. S. Grubbs, C. T. Dewberry, K. C. Etchison, K. E. Kerr and S. A. Cooke, Rev. Sci. Instrum., 2007, 78, 096106.

19 D. A. Obenchain, A. A. Elliot, A. L. Steber, R. A. Peebles, S. A. Peebles, C. J. Wurrey and G. A. Guirgis, J. Mol. Spectrosc., 2010, 261, 3.

20 M. C. McCarthy, W. Chen, M. J. Travers and P. Thaddeus, Astrophys. J., Suppl. Ser., 2000, 129, 611.

21 C. Pérez, S. Mata, S. Blanco, J. C. López and J. L. Alonso, J. Phys. Chem. A, 2011, 115, 9653.

22 S. L. Miller, Science, 1953, 117, 528.

23 S. L. Miller and H. C. Urey, Science, 1959, 130, 245.

24 F. Bloch, Phys. Rev., 1946, 70, 460.

25 S. J. Henley, J. D. Carey, S. R. P. Silva, G. M. Fuge, M. N. R. Ashfold and D. Anglos, Phys. Rev. B: Condens. Matter Mater. Phys., 2005, 72, 205413.

26 N. A. Seifert, D. P. Zaleski, C. Pérez, J. L. Neill, B. H. Pate, M. Vallejo-López, A. Lessarri, E. J. Cocinero, F. Castaño and I. Kleiner, Angew. Chem., Int. Ed., 2014, 53, 3210.

27 N. A. Seifert, A. L. Steber, J. L. Neill, C. Pérez, D. P. Zaleski, B. H. Pate and A. Lesarri, Phys. Chem. Chem. Phys., 2013, 15, 11468.

28 J. A. Kroll, S. T. Shipman and S. L. Widicus Weaver, J. Mol. Spectrosc., 2014, 295, 52.
29 H. V. L. Nguyen, I. Kleiner, S. T. Shipman, Y. Mae, K. Hirose, S. Hatanaka and K. Kobayashi, J. Mol. Spectrosc., 2014, 299, 17.

30 S. Mata, I. Peña, C. Cabezas, J. C. López and J. L. Alonso, J. Mol. Spectrosc., 2012, 280, 91.

31 T. D. Jaeger, D. van Heijnsbergen, S. J. Klippenstein, G. von Helden, G. Meijer and M. A. Duncan, J. Am. Chem. Soc., 2004, 126, 10981.

32 A. Thoma, B. E. Wurfel, R. Schlachta and G. M. Lask, J. Phys. Chem., 1992, 96, 7231.

33 H. Phillips, S. Kubodera, R. Sauerbrey, F. K. Tittel and P. J. Wisoff, IEEE J. Quantum Electron., 1991, 27, 95.

34 M. A. Buntine, D. C. McGlivery and R. J. S. Morrison, J. Mass Spectrom., 1991, 26, 195.

35 K. D. Hensel, C. Styger, W. Jäger, A. J. Merer and M. C. L. Gerry, J. Chem. Phys., 1993, 99, 3320.

36 S. G. Batten and A. C. Legon, Chem. Phys. Lett., 2006, 422, 192.

37 K. A. Walker and M. C. L. Gerry, J. Mol. Spectrosc., 1997, $182,178$.

38 V. A. Mikhailov, F. J. Roberts, S. L. Stephens, S. J. Harris, D. P. Tew, J. N. Harvey, N. R. Walker and A. C. Legon, J. Chem. Phys., 2011, 134, 134305.

39 S. L. Stephens, D. P. Tew, N. R. Walker and A. C. Legon, J. Mol. Spectrosc., 2011, 267, 163.

40 S. L. Stephens, W. Mizukami, D. P. Tew, N. R. Walker and A. C. Legon, J. Chem. Phys., 2012, 136, 064306.

41 S. L. Stephens, N. R. Walker and A. C. Legon, J. Chem. Phys., 2011, 135, 224309.

42 S. G. Batten, A. G. Ward and A. C. Legon, J. Mol. Spectrosc., 2006, 780-781, 300.

43 S. Z. Riaz, S. L. Stephens, W. Mizukami, D. P. Tew, N. R. Walker and A. C. Legon, Chem. Phys. Lett., 2012, 531, 1. 44 S. L. Stephens, D. M. Bittner, V. A. Mikhailov, W. Mizukami, D. P. Tew, N. R. Walker and A. C. Legon, Inorg. Chem., 2014, 53, 10722.

45 S. L. Stephens, D. P. Tew, N. R. Walker and A. C. Legon, J. Chem. Phys., 2011, 135, 224309.

46 G. S. Grubbs, D. J. Frohman, S. E. Novick and S. A. Cooke, J. Mol. Spectrosc., 2012, 280, 85.

47 S. L. Stephens, D. P. Zaleski, W. Mizukami, D. P. Tew, N. R. Walker and A. C. Legon, J. Chem. Phys., 2014, 140, 124310.

48 J. M. Hollis, A. J. Remijan, P. R. Jewell, F. J. Lovas, GBT PRIMOS, http://www.cv.nrao.edu/ aremijan/PRIMOS/.

49 M. Guélin, J. Cernicharo, M. J. Travers, M. C. McCarthy, C. A. Gottlieb, P. Thaddeus, M. Ohishi, S. Saito and S. Yamamoto, Astron. Astrophys., 1997, 317, L1.

50 M. J. Travers, M. C. McCarthy, P. Kalmus, C. A. Gottlieb and P. Thaddeus, Astrophys. J., 1996, 469, L65.

51 Y. Ohshima and Y. Endo, Chem. Phys. Lett., 1996, 256, 635. 52 C. A. Gottlieb, A. J. Apponi, M. C. McCarthy and P. Thaddeus, J. Chem. Phys., 2000, 113, 1910.

53 S. Brünken, H. Gupta, C. A. Gottlieb, M. C. McCarthy and P. Thaddeus, Astrophys. J., 2007, 644, L43.

54 M. C. McCarthy, P. Thaddeus, J. J. Wilke and H. F. Schaefer III, J. Chem. Phys., 2009, 130, 234304. 
55 J. L. Neill, A. L. Steber, M. T. Muckle, D. P. Zaleski, V. Lattanzi, S. Spezzano, M. C. McCarthy, A. J. Remijan, D. N. Friedel, S. L. Widicus Weaver and B. H. Pate, J. Phys. Chem. A, 2011, $115,6472$.

56 J. L. Neill, M. T. Muckle, D. P. Zaleski, A. L. Steber, B. H. Pate, V. Lattanzi, S. Spezzano, M. C. McCarthy and A. J. Remijan, Astrophys. J., 2012, 755, 153.

57 D. T. Halfen, L. M. Ziurys, M. C. McCarthy, C. A. Gottleib and P. Thaddeus, Astrophys. J., 2009, 702, L124.

58 www.splatalogue.net.

59 CDMS: H. S. P. Müller, F. Schlöder, J. Stutzki and G. Winnewisser, J. Mol. Struct., 2005, 742, 215.

60 R. A. Loomis, D. P. Zaleski, A. L. Steber, J. L. Neill, M. T. Muckle, B. J. Harris, J. M. Hollis, P. R. Jewell, V. Lattanzi, F. J. Lovas, O. Martinez Jr., M. C. McCarthy, A. J. Remijan, B. H. Pate and J. F. Corby, Astrophys. J., 2013, 765, L9.

61 D. P. Zaleski, N. A. Seifert, A. L. Steber, M. T. Muckle, R. A. Loomis, J. F. Corby, O. Martinez Jr., K. N. Crabtree, P. R. Jewell, J. M. Hollis, F. J. Lovas, D. Vasquez, J. Nyiramahirwe, N. Sciortino, K. Johnson, M. C. McCarthy, A. J. Remijan and B. H. Pate, Astrophys. J., 2013, 765, L10.

62 P. D. Godfrey, R. D. Brown, B. J. Robinson and M. W. Sinclair, Astrophys. J., 1973, 13, L119.

63 M. C. McCarthy and P. Thaddeus, Chem. Soc. Rev., 2001, 30, 177. 64 J. E. Elsila, J. P. Dworkin, M. P. Bernstein, M. P. Martin and S. A. Sandford, Astrophys. J., 2007, 660, 911.

65 J. Oró, Nature, 1961, 191, 1193.

66 J. P. Ferris and L. E. Orgel, J. Am. Chem. Soc., 1966, 88, 1074. 67 J. P. Ferris, P. C. Joshi, E. H. Edelson and J. G. Lawless, J. Mol. Evol., 1978, 11, 293.

68 R. Sanchez, J. P. Ferris and L. E. Orgel, Science, 1966, 153, 72. 69 A. W. Schwartz, H. Joosten and A. B. Voet, BioSystems, 1982, 15, 191.

70 E. Borquez, H. J. Cleaves, A. Lazcano and S. L. Miller, Origins Life Evol. Biospheres, 2005, 35, 79.
71 D. Roy, K. Najafian and P. von Ragué Schleyer, Proc. Natl. Acad. Sci. U. S. A., 2007, 104, 17272.

72 W. Gilbert, Nature, 1986, 319, 618.

73 K. N. Crabtree, O. Martinez Jr. and M. C. McCarthy, J. Phys. Chem. A, 2014, 117, 11282.

74 D. S. N. Parker, A. V. Wilson, R. I. Kaiser, T. Labrador and A. M. Mebel, J. Am. Chem. Soc., 2012, 134, 13896.

75 K. J. Yacoubi, J. Electrochem. Soc., 1999, 146, 3009.

76 S.-W. Hu, Y. Wang, X.-Y. Wang, T.-W. Chu and X.-Q. Liu, J. Phys. Chem. A, 2003, 107, 9189.

77 S. Koseki and A. J. Ishitani, Appl. Phys., 1992, 72, 5808.

78 H. E. O’Neal, M. Ring, J. G. Martin and M. T. Navio, J. Phys. Chem. A, 1998, 102, 8493.

79 A. A. Bagatur'yants, K. P. Novoselov, A. A. Safonov, J. Vernon Cole, M. Stoker and A. A. Korkin, Surf. Sci., 2001, 486, 213.

80 C. Karunatlaka, A. J. Shirar, G. L. Storck, K. M. Hotopp, E. B. Biddle, R. Crawley Jr. and B. C. Dian, J. Phys. Chem. Lett., 2010, 1, 1547.

81 A. Lifshitz and M. Bidani, J. Phys. Chem., 1989, 93, 1139.

82 A. Burcat, Fuel, 1975, 54, 87.

83 G. S. Grubbs and S. A. Cooke, J. Mol. Spectrosc., 2010, 259, 120.

84 H.-J. Kockert, D. P. Zaleski, S. L. Stephens, L.-M. Dickens, C. J. Evans, N. R. Walker, 2014, arXiv:1408.2276 [physics.chem-ph].

85 N. S. Dattani, L. N. Zack, M. Sun, E. R. Johnson, R. J. Le Roy, L. M. Ziurys, unpublished work.

86 J. Liu, J. Dai, S. L. Chin and X.-C. Zhang, Nat. Photonics, 2010, 4, 627.

87 D. K. Killinger and N. Menyuk, Science, 1987, 235, 37.

88 D. Stramski, R. A. Reynolds, M. Kahru and B. G. Mitchell, Science, 1999, 285, 239.

89 N. M. Kidwell, V. Vaquero-Vara, T. K. Ormond, G. T. Buckingham, D. Zhang, D. N. Mehta-Hurt, L. McCaslin, M. R. Nimlos, J. W. Daily, B. C. Dian, J. F. Stanton, G. B. Ellison and T. S. Zwier, J. Phys. Chem. Lett., 2014, 5, 2201. 